\title{
Nondecoupling phenomena in QED in a magnetic field and noncommutative QED
}

\author{
E.V. Gorbar ${ }^{*}$ Michio Hashimoto \\ Department of Applied Mathematics, University of Western Ontario, London, Ontario N6A 5B7, Canada
}

(Dated: November 7, 2018)

\begin{abstract}
The dynamics in QED in a strong constant magnetic field and its connection with the noncommutative QED are studied. It is shown that in the regime with the lowest Landau level (LLL) dominance the $U(1)$ gauge symmetry in the fermion determinant is transformed into the noncommutative $U(1)_{n c}$ gauge symmetry. In this regime, the effective action is intimately connected with that in noncommutative QED and the original $U(1)$ gauge Ward identities are broken (the LLL anomaly). On the other hand, it is shown that although a contribution of each of an infinite number of higher Landau levels is suppressed in an infrared region, their cumulative contribution is not (a nondecoupling phenomenon). This leads to a restoration of the original $U(1)$ gauge symmetry in the infrared dynamics. The physics underlying this phenomenon reflects the important role of a boundary dynamics at spatial infinity in this problem.
\end{abstract}

PACS numbers: 11.10.Nx, 11.15.-q, 12.20.-m

Since the classical papers 1, 2], the problem of QED in a constant magnetic field has been thoroughly studied (for a recent review, see Ref. [3] ). In this letter, we consider this problem for the case of a strong magnetic field. In particular, we study the connection of this dynamics with that in noncommutative QED (for reviews of noncommutative field theories (NCFT), see Ref. [4]). The motivation for this study was the recent results obtained in Ref. [5], where the connection between the dynamics in the Nambu-Jona-Lasinio (NJL) model in a strong magnetic field and that in NCFT was established. The main conclusion of that paper was that although in the regime with the lowest Landau level (LLL) dominance the NJL model determines a NCFT, this NCFT is different from the conventional ones considered in the literature. In particular, the UV/IR mixing, taking place in the conventional NCFT [6], is absent in this case. The reason of that is an inner structure (i.e., dynamical form-factors) of neutral composites in this model.

In this letter, some sophisticated features of the dynamics in QED in a strong magnetic field are revealed. It is shown that in the approximation with the lowest Landau level (LLL) dominance, the initial $U(1)$ gauge symmetry in the fermion determinant is transformed into the noncommutative $U(1)_{n c}$ gauge symmetry. In this regime, the effective action is intimately connected with that in noncommutative QED and the original $U(1)$ gauge Ward identities are broken (we call this phenomenon an LLL anomaly). In fact, this dynamics yields a modified noncommutative QED in which the UV/IR mixing is absent, similarly to the case of the NJL model in a strong magnetic field [5]. The reason of that is an inner structure (i.e., a dynamical form-factor) of photons in a strong magnetic field. However, it is not the end of the story. We show that adding the contribution of all the higher Landau levels removes the LLL anomaly and restores the original $U(1)$ gauge symmetry. This restoration happens in a quite sophisticated way: although a contribution of each of an infinite number of higher Landau levels is suppressed in an infrared region, their cumulative contribution is not (a nondecoupling phenomenon). As will be discussed below, this phenomenon reflects the important role of a boundary dynamics at spatial infinity in this problem. We also indicate the kinematic region where the LLL approximation is reliable.

To put the dynamics in QED in a magnetic field under control, we will consider the case with a large number of fermion flavors $N$, when the $1 / N$ expansion is reliable. We also choose the current fermion mass $m$ satisfying the condition $m_{d y n} \ll m \ll \sqrt{|e B|}$, where $m_{d y n}$ is the dynamical mass of fermions generated in the chiral symmetric QED in a magnetic field [7]. ${ }^{1}$ The condition $m_{d y n} \ll m$ guarantees that there are no light (pseudo) Nambu-Goldstone bosons, and the only particles in the low energy effective theory in this model are photons. As to the condition $m \ll \sqrt{|e B|}$, it implies that the magnetic field is very strong.

Integrating out fermions, we obtain the effective action for photons in the leading order in $1 / N$ :

$$
\Gamma=\Gamma^{(0)}+\Gamma^{(1)}, \quad \Gamma^{(0)}=-\frac{1}{4} \int d^{4} x f_{\mu \nu}^{2}, \quad \Gamma^{(1)}=-i N \operatorname{Tr} \operatorname{Ln}\left[i \gamma^{\mu}\left(\partial_{\mu}-i e A_{\mu}\right)-m\right],
$$

\footnotetext{
*Electronic address: egorbar@uwo.ca On leave from Bogolyubov Institute for Theoretical Physics, 03143, Kiev, Ukraine

${ }^{\dagger}$ Electronic address: mhashimo@uwo.ca

${ }_{\ddagger}^{\ddagger}$ Electronic address: vmiransk@uwo.ca On leave from Bogolyubov Institute for Theoretical Physics, 03143, Kiev, Ukraine

1 The dynamical mass is $m_{d y n} \simeq \sqrt{|e B|} \exp (-N)$ for a large running coupling $\tilde{\alpha}_{b} \equiv N \alpha_{b}$ related to the magnetic scale $\sqrt{|e B|}$, and $m_{d y n} \sim \sqrt{|e B|} \exp \left[-\frac{\pi N}{\tilde{\alpha}_{b} \ln \left(1 / \tilde{\alpha}_{b}\right)}\right]$ when the coupling $\tilde{\alpha}_{b}$ is weak 7$]$.
} 
where $f^{\mu \nu}=\partial_{\mu} A_{\nu}-\partial_{\nu} A_{\mu}$ and the vector field $A_{\mu}=A_{\mu}^{c l}+\tilde{A}_{\mu}$, where the classical part $A_{\mu}^{c l}$ is $A_{\mu}^{c l}=\left\langle 0\left|A_{\mu}\right| 0\right\rangle$. Since the constant magnetic field $B$ is a solution of the exact equations in QED (see for example the discussion in Sec. 2 in the second paper in Ref. [7]), it is

$$
A_{\mu}^{c l}=\left(0, \frac{B x^{2}}{2},-\frac{B x^{1}}{2}, 0\right) .
$$

This field describes a constant magnetic field directed in the $+x^{3}$ direction and we use the so called symmetric gauge for $A_{\mu}^{c l}$.

For a strong magnetic field $|e B| \gg m^{2}$, it is naturally to expect that in the infrared region with momenta $k \ll \sqrt{|e B|}$, the LLL approximation should be reliable. Indeed, let us consider the fermion propagator in a magnetic field [2]:

$$
S(x, y)=\exp \left[\frac{i e}{2}(x-y)^{\mu} A_{\mu}^{\mathrm{ext}}(x+y)\right] \tilde{S}(x-y),
$$

where the Fourier transform of the translationally invariant part $\tilde{S}$ can be decomposed over the Landau levels [8]:

$$
\tilde{S}(k)=i \exp \left(-\frac{\boldsymbol{k}_{\perp}^{2}}{|e B|}\right) \sum_{n=0}^{\infty}(-1)^{n} \frac{D_{n}(e B, k)}{k_{\|}^{2}-m^{2}-2|e B| n}
$$

with $\boldsymbol{k}_{\perp} \equiv\left(k^{1}, k^{2}\right)$ and $k_{\|} \equiv\left(k_{0}, k_{3}\right)$. The functions $D_{n}(e B, k)$ are expressed through the generalized Laguerre polynomials $L_{m}^{\alpha}$ :

$$
\begin{aligned}
D_{n}(e B, k)= & \left(k_{\|} \gamma^{\|}+m\right)\left[\left(1-i \gamma^{1} \gamma^{2} \operatorname{sign}(e B)\right) L_{n}\left(2 \frac{\boldsymbol{k}_{\perp}^{2}}{|e B|}\right)-\left(1+i \gamma^{1} \gamma^{2} \operatorname{sign}(e B)\right) L_{n-1}\left(2 \frac{\boldsymbol{k}_{\perp}^{2}}{|e B|}\right)\right] \\
& +4\left(k^{1} \gamma^{1}+k^{2} \gamma^{2}\right) L_{n-1}^{1}\left(2 \frac{\boldsymbol{k}_{\perp}^{2}}{|e B|}\right),
\end{aligned}
$$

where $\gamma^{\|} \equiv\left(\gamma^{0}, \gamma^{3}\right)$. Relation (44) seems to suggest that in the infrared region, with $k_{\perp}, k_{\|} \ll \sqrt{|e B|}$, all the higher Landau levels with $n \geq 1$ decouple and only the LLL with $n=0$ is relevant. Although this argument is physically convincing, there may be a potential flaw due to an infinite number of the Landau levels. As will be shown below, this is indeed the case in this problem: the cumulative contribution of the higher Landau levels does not decouple.

But first we will consider the dynamics in the LLL approximation. In this case, the calculation of the effective action (11) is reduced to calculating fermion loops with the LLL fermion propagators. Such a problem in the NJL model in a magnetic field has been recently solved in Ref. [5]. The extension of that analysis to the case of QED is straightforward. The effective action (1) in the LLL approximation is given by

$$
\Gamma_{L L L}=\Gamma^{(0)}+\Gamma_{L L L}^{(1)}, \quad \Gamma_{L L L}^{(1)}=-\frac{i N|e B|}{2 \pi} \int d^{2} x_{\perp} \operatorname{Tr}_{\|}\left[\mathcal{P} \operatorname{Ln}\left[i \gamma^{\|}\left(\partial_{\|}-i e \mathcal{A}_{\|}\right)-m\right]\right]_{*}
$$

(compare with Eq. (54) in [5]). Here $*$ is the symbol of the Moyal star product, which is a signature of a NCFT [4], the projector $\mathcal{P}$ is $\mathcal{P} \equiv\left[1-i \gamma^{1} \gamma^{2} \operatorname{sign}(e B)\right] / 2$, and the longitudinal "smeared" fields $\mathcal{A}_{\|}$are defined as $\mathcal{A}_{\|}=e^{\frac{\nabla^{2}}{4 \mid e B}} A_{\|}$ [5], where $\nabla_{\perp}^{2}$ is the transverse Laplacian. Notice that $\mathcal{P}$ is the projector on the fermion (antifermion) states with the spin polarized along (opposite to) the magnetic field and that the one-loop term $\Gamma_{L L L}^{(1)}$ in (6) includes only the longitudinal field $\mathcal{A}_{\|}=\left(\mathcal{A}_{0}, \mathcal{A}_{3}\right)$. This is because the LLL fermions couple only to the longitudinal components of the photon field [7].

In action (6), the trace $\operatorname{Tr}_{\|}$, related to the longitudinal subspace, is taken in the functional sense and the star product relates to the space transverse coordinates. Therefore the LLL dynamics determines a NCFT with noncommutative transverse coordinates $\hat{x}_{\perp}^{a}, a=1,2$ :

$$
\left[\hat{x}_{\perp}^{a}, \hat{x}_{\perp}^{b}\right]=i \frac{1}{e B} \epsilon^{a b} \equiv i \theta^{a b} .
$$

The structure of the logarithm of the fermion determinant in $\Gamma_{L L L}^{(1)}$ (66) implies that it is invariant not under the initial $U(1)$ gauge symmetry but under the noncommutative $U(1)_{n c}$ gauge one [4] (henceforth we omit the subscript || in gauge fields):

$$
\begin{array}{ll}
\mathcal{A}_{\mu} \rightarrow U(x) * \mathcal{A}_{\mu} * U^{-1}(x)+\frac{i}{e} U(x) * \partial_{\mu} U^{-1}(x), & (\mu=0,3) \\
\mathcal{F}_{\mu \nu} \rightarrow U(x) * \mathcal{F}_{\mu \nu} * U^{-1}(x), & (\mu, \nu=0,3)
\end{array}
$$


where $U(x)=\left(e^{i \lambda(x)}\right)_{*}$ and the field strength $\mathcal{F}_{\mu \nu}$ is

$$
\mathcal{F}_{\mu \nu}=\partial_{\mu} \mathcal{A}_{\nu}-\partial_{\nu} \mathcal{A}_{\mu}-i e\left[\mathcal{A}_{\mu}, \mathcal{A}_{\nu}\right]_{\mathrm{MB}}
$$

with the Moyal bracket

$$
\left[\mathcal{A}_{\mu}, \mathcal{A}_{\nu}\right]_{\mathrm{MB}} \equiv \mathcal{A}_{\mu} * \mathcal{A}_{\nu}-\mathcal{A}_{\nu} * \mathcal{A}_{\mu}
$$

Therefore the derivative expansion of $\Gamma_{L L L}^{(1)}$ should be expressed through terms with the star product of the field $\mathcal{F}_{\mu \nu}$ and its covariant derivatives:

$$
\Gamma_{L L L}^{(1)}=a_{0} S_{\mathcal{F}^{2}}+a_{1} S_{\mathcal{F}^{3}}+a_{2} S_{(\mathcal{D F})^{2}}+a_{3} S_{\mathcal{D}^{2} \mathcal{F}^{2}}+\cdots
$$

where

$$
\begin{aligned}
S_{\mathcal{F}^{2}} & \equiv-\frac{1}{4} \int d^{2} x_{\perp} d^{2} x_{\|} \mathcal{F}_{\mu \nu} * \mathcal{F}^{\mu \nu}, & S_{\mathcal{F}^{3}} \equiv i e \int d^{2} x_{\perp} d^{2} x_{\|} \mathcal{F}_{\mu \nu} * \mathcal{F}^{\nu \lambda} * \mathcal{F}_{\lambda}{ }^{\mu}, \\
S_{(\mathcal{D} \mathcal{F})^{2}} & \equiv \int d^{2} x_{\perp} d^{2} x_{\|} \mathcal{D}_{\lambda} \mathcal{F}^{\lambda \mu} * \mathcal{D}^{\rho} \mathcal{F}_{\rho \mu}, & S_{\mathcal{D}^{2} \mathcal{F}^{2}} \equiv \int d^{2} x_{\perp} d^{2} x_{\|} \mathcal{D}_{\lambda} \mathcal{F}_{\mu \nu} * \mathcal{D}^{\lambda} \mathcal{F}^{\mu \nu}
\end{aligned}
$$

and the covariant derivative of $\mathcal{F}_{\mu \nu}$ is $\mathcal{D}_{\lambda} \mathcal{F}_{\mu \nu}=\partial_{\lambda} \mathcal{F}_{\mu \nu}-i e\left[\mathcal{A}_{\lambda}, \mathcal{F}_{\mu \nu}\right]_{\mathrm{MB}}$. These are all independent operators which have the dimension four and six. In particular, by using the Jacobi identity,

$$
\left[\mathcal{D}_{\mu},\left[\mathcal{D}_{\nu}, \mathcal{D}_{\lambda}\right]_{\mathrm{MB}}\right]_{\mathrm{MB}}+\left[\mathcal{D}_{\nu},\left[\mathcal{D}_{\lambda}, \mathcal{D}_{\mu}\right]_{\mathrm{MB}}\right]_{\mathrm{MB}}+\left[\mathcal{D}_{\lambda},\left[\mathcal{D}_{\mu}, \mathcal{D}_{\nu}\right]_{\mathrm{MB}}\right]_{\mathrm{MB}}=0
$$

and the relation $\mathcal{F}_{\mu \nu}=i e^{-1}\left[\mathcal{D}_{\mu}, \mathcal{D}_{\nu}\right]_{\mathrm{MB}}$, one can easily check that the operator $\int d^{2} x_{\perp} d^{2} x_{\|} \mathcal{D}_{\lambda} \mathcal{F}_{\mu \nu} * \mathcal{D}^{\mu} \mathcal{F}^{\nu \lambda}$ is not independent: it is equal to $-1 / 2 S_{\mathcal{D}^{2} \mathcal{F}^{2}}$.

The coefficients $a_{i},(i=0,1,2,3, \cdots)$ in Eq. (11) can be found from the $n$-point photon vertices

$$
T_{L L L}^{(n)}=i \frac{(i e)^{n} N|e B|}{2 \pi n} \int d^{2} x^{\perp} d^{2} x_{1}^{\|} \cdots d^{2} x_{n}^{\|} \operatorname{tr}\left[S_{\|}\left(x_{1}^{\|}-x_{2}^{\|}\right) \hat{\mathcal{A}}\left(x^{\perp}, x_{2}^{\|}\right) \ldots S_{\|}\left(x_{n}^{\|}-x_{1}^{\|}\right) \hat{\mathcal{A}}\left(x^{\perp}, x_{1}^{\|}\right)\right]_{*}
$$

by expanding the vertices in powers of external momenta (here $S_{\|}\left(x_{\|}\right)=\int \frac{d^{2} k_{\|}}{(2 \pi)^{2}} e^{-i k_{\|} x^{\|}} \frac{i}{k_{\|} \gamma^{\|}-m} \mathcal{P}$ and $\left.\hat{\mathcal{A}} \equiv \gamma^{\|} \mathcal{A}_{\|}\right)$. In particular, from the vertices $T_{L L L}^{(2)}$ and $T_{L L L}^{(3)}$, we find the coefficients $a_{0}, a_{1}, a_{2}$, and $a_{3}$ connected with the operators of the dimension four and six in the derivative expansion (11) of $\Gamma_{L L L}^{(1)}$ :

$$
a_{0}=\frac{\tilde{\alpha}}{3 \pi} \frac{|e B|}{m^{2}}, \quad a_{1}=\frac{1}{60 m^{2}} a_{0}, \quad a_{2}=-\frac{1}{10 m^{2}} a_{0}, \quad a_{3}=0,
$$

where $\tilde{\alpha} \equiv N \alpha=N e^{2} /(4 \pi)$ (since in the presence of a magnetic field the charge conjugation symmetry is broken, the 3 -point vertex is nonzero).

The $U(1)$ gauge Ward identities imply that the $n$-point photon amplitude $T^{\mu_{1} \ldots \mu_{n}}\left(x_{1}, \ldots, x_{n}\right)$ should be transverse, i.e., $\partial_{\mu_{i}} T^{\mu_{1} \ldots \mu_{n}}\left(x_{1}, \ldots, x_{n}\right)=0$. It is easy to show that the 2 -point vertex $T_{L L L}^{\mu_{1} \mu_{2}}$ yielding the polarization operator is transverse indeed. Now let us turn to the 3-point vertex and show that it is not transverse, i.e., the Ward identities connected with the initial gauge $U(1)$ are broken in the LLL approximation. In the momentum space, the vertex is

$$
T_{L L L}^{\mu_{1} \mu_{2} \mu_{3}}\left(k_{1}, k_{2}, k_{3}\right)=N e^{3} \frac{|e B|}{2 \pi} \sin \left(\frac{1}{2} \theta_{a b} k_{1 \perp}^{a} k_{2 \perp}^{b}\right) \Delta_{L L L}^{\mu_{1 \|} \mu_{2 \|} \mu_{3 \|}}\left(k_{1 \|}, k_{2 \|}, k_{3 \|}\right)
$$

with $^{2}$

$$
\Delta_{L L L}^{\mu_{1 \|} \mu_{2 \|} \mu_{3 \|}}\left(k_{1 \|}, k_{2 \|}, k_{3 \|}\right) \equiv \int \frac{d^{2} \ell_{\|}}{i(2 \pi)^{2}} \frac{\operatorname{tr}\left[\gamma_{\|}^{\mu_{1}}\left[\left(\ell-\not k_{1}\right)_{\|}+m\right] \gamma_{\|}^{\mu_{2}}\left[\left(\ell+\not k_{3}\right)_{\|}+m\right] \gamma_{\|}^{\mu_{3}}\left(\ell_{\|}+m\right)\right]}{\left(\ell_{\|}^{2}-m^{2}\right)\left[\left(\ell-k_{1}\right)_{\|}^{2}-m^{2}\right]\left[\left(\ell+k_{3}\right)_{\|}^{2}-m^{2}\right]} .
$$

\footnotetext{
2 The explicit expression for $\Delta_{L L L}^{\mu_{1 \|} \mu_{2 \|} \mu_{3 \|}}$ is given in terms of the two dimensional version of the Passarino-Veltman functions [9]. It is quite cumbersome and will be written down elsewhere.
} 
The argument of the sine in Eq. (16) is the Moyal cross product with $\theta_{a b}=\epsilon^{a b} / e B$ (see Eq. (77)). It is easy to find that the transverse part of the vertex (16) is not zero and equal to

$$
k_{1 \mu_{1}} T_{L L L}^{\mu_{1} \mu_{2} \mu_{3}}\left(k_{1}, k_{2}, k_{3}\right)=-\frac{2 e}{i} \sin \left(\frac{1}{2} \theta_{a b} k_{1 \perp}^{a} k_{2 \perp}^{b}\right)\left[\Pi_{\|}^{\mu_{2} \mu_{3}}\left(k_{2 \|}\right)-\Pi_{\|}^{\mu_{2} \mu_{3}}\left(k_{3 \|}\right)\right],
$$

with

$$
\Pi_{\|}^{\mu \nu}\left(k_{\|}\right)=i \frac{2 \tilde{\alpha}|e B|}{\pi}\left(g_{\|}^{\mu \nu}-\frac{k_{\|}^{\mu} k_{\|}^{\nu}}{k_{\|}^{2}}\right) \Pi\left(k_{\|}^{2}\right), \quad \Pi\left(k_{\|}^{2}\right) \equiv 1+\frac{2 m^{2}}{k_{\|}^{2} \sqrt{1-\frac{4 m^{2}}{k_{\|}^{2}}}} \ln \frac{1+\sqrt{1-\frac{4 m^{2}}{k_{\|}^{2}}}}{-1+\sqrt{1-\frac{4 m^{2}}{k_{\|}^{2}}}} .
$$

Here we defined $g_{\|}=\operatorname{diag}(1,-1)$ [note that $\Pi_{\|}^{\mu \nu}$ coincides with the polarization tensor in the $(1+1)$-dimensional QED if the parameter $2 \tilde{\alpha}|e B|$ here is replaced by the coupling $e_{1}^{2}$ in $\left.\mathrm{QED}_{1+1}\right]$. Thus, the original $U(1)$ gauge Ward identities are broken in the LLL approximation. We will call this an LLL anomaly.

Note that all the vertices $T_{L L L}^{(n)}$ with $n \geq 3$ are finite and a logarithmic divergence in the vertex $T_{L L L}^{(2)}$, which is proportional to the polarization operator, is absent if one uses a gauge invariant regularization. In fact, it is sufficient if the regularization is invariant under the longitudinal $U(1)_{\|}$gauge group with phase parameters $\alpha\left(x^{\|}\right)$depending only on longitudinal coordinates. This $U(1)_{\|}$is a subgroup of both the gauge $U(1)$ and the noncommutative gauge $U(1)_{n c}$ and it is the gauge symmetry of the whole action $\Gamma_{L L L}$ (6). Indeed, while the free Maxwell term $\Gamma^{(0)}$ in (6) is invariant under the gauge $U(1)$, the one-loop term $\Gamma_{L L L}^{(1)}$ is invariant under the $U(1)_{n c}$. Another noticeable point is that the divergence (18) is not a polynomial function of momenta and have branch point singularities. Therefore the fact that $T_{L L L}^{(3)}$ is not transverse is regularization independent.

The origin of the LLL anomaly is clear: the $T_{L L L}^{(n)}$ vertices come from the one-loop part $\Gamma_{L L L}^{(1)}$ of the action which is invariant not under the gauge $U(1)$ but under the $U(1)_{n c}$. Therefore the Ward identities for the vertices $T_{L L L}^{(n)}$ reflect not the $U(1)$ gauge symmetry but the noncommutative symmetry $U(1)_{n c}$.

Notice that the action $\Gamma_{L L L}$ (6) determines a conventional noncommutative QED only in the case of an induced photon field, when the Maxwell term $\Gamma^{(0)}$ is absent. When this term is present, the action also determines a NCFT, however, this NCFT is different from the conventional ones considered in the literature. In particular, expressing the photon field $A_{\mu}$ through the smeared field $\mathcal{A}_{\mu}$ as $A_{\mu}=e^{\frac{-\nabla_{\perp}^{2}}{4|e B|}} \mathcal{A}_{\mu}$, we find that the propagator of the smeared field rapidly, as $e^{\frac{-p_{1}^{2}}{2|e B|}}$, decreases for large transverse momenta. The form-factor $e^{\frac{-p_{1}^{2}}{2|e B|}}$ built in the smeared field reflects an inner structure of photons in a magnetic field. This feature leads to removing the UV/IR mixing in this NCFT (compare with the analysis of the UV/IR mixing in Sec. 4 of Ref. [5]).

It is clear however that, since the initial QED has the usual $U(1)$ gauge symmetry, there should exist an additional contribution that restores the $U(1)$ Ward identities broken in the LLL approximation. We will show that this contribution comes from heavy (naively decoupled) higher Landau levels (HLL), and it is necessary to consider the contribution of all of them in order to restore the Ward identities.

Let us consider the 3-point vertex replacing one of the LLL propagator by the full one in Eq. (4). We find that at each $n \geq 1$ the contributions in the vertex of the first, second and third terms in Eq. (5) are respectively

$$
\begin{aligned}
\Delta_{H L L}^{\mu_{\|} \nu_{\|} \lambda_{\|}}(p, q, k) & \sim \frac{(-1)^{n}}{n|e B|} f_{\|}^{\mu \nu \lambda}\left(p_{\|}, q_{\|}, k_{\|}\right) \\
\Delta_{H L L}^{\mu_{\perp} \nu_{\perp} \lambda_{\|}}(p, q, k) & \sim \frac{(-1)^{n}}{n|e B|}\left[g_{\perp}^{\mu \nu} h_{1}\left(p_{\perp}, q_{\perp}, k_{\perp}\right)+\epsilon_{\perp}^{\mu \nu} h_{2}\left(p_{\perp}, q_{\perp}, k_{\perp}\right) \operatorname{sign}(e B)\right]\left(q_{\|}^{\lambda}-\frac{(q \cdot k)_{\|}}{k_{\|}^{2}} k_{\|}^{\lambda}\right) \Pi\left(k_{\|}^{2}\right), \\
\Delta_{H L L}^{\mu_{\perp} \nu_{\|} \lambda_{\|}}(p, q, k) & \sim \frac{(-1)^{n}}{n|e B|} h_{3}^{\mu_{\perp}}\left(p_{\perp}, q_{\perp}, k_{\perp}\right)\left(g_{\|}^{\nu \lambda}-\frac{k_{\|}^{\nu} k_{\|}^{\lambda}}{k_{\|}^{2}}\right) \Pi\left(k_{\|}^{2}\right),
\end{aligned}
$$

where $g_{\perp}=\operatorname{diag}(-1,-1),(p \cdot q)_{\|}=p^{0} q^{0}-p^{3} q^{3}$, and $f_{\|}^{\mu \nu \lambda}, h_{1,2}$ and $h_{3}^{\mu_{\perp}}$ are some smooth functions of longitudinal and transverse momenta. As was expected, the contribution of each of the individual HLL with $n \geq 1$ is suppressed by powers of $1 /|e B|$ in the infrared region. It is however quite remarkable that despite the suppression of individual HLL contributions, their cumulative contribution is not suppressed in the infrared region (a nondecoupling effect). In 
fact, using the relation 10$]$

$$
(1-z)^{-(\alpha+1)} \exp \left(\frac{x z}{z-1}\right)=\sum_{n=0}^{\infty} L_{n}^{\alpha}(x) z^{n}
$$

and integrating it with respect to $z$, we can perform explicitly the summation over the HLL contributions and obtain the 3-point vertex that satisfies the Ward identities for the $U(1)$ gauge symmetry:

$$
T^{\mu_{1} \mu_{2} \mu_{3}}\left(k_{1}, k_{2}, k_{3}\right)=T_{L L L}^{\mu_{1} \mu_{2} \mu_{3}}\left(k_{1}, k_{2}, k_{3}\right)+T_{H L L 1}^{\mu_{1} \mu_{2} \mu_{3}}\left(k_{1}, k_{2}, k_{3}\right)+T_{H L L 2}^{\mu_{1} \mu_{2} \mu_{3}}\left(k_{1}, k_{2}, k_{3}\right),
$$

where

$$
\begin{aligned}
& T_{H L L 1}^{\mu_{1} \mu_{2} \mu_{3}}\left(k_{1}, k_{2}, k_{3}\right)=\frac{2 e}{i} \sin \left(\frac{1}{2} \theta_{a b} k_{1 \perp}^{a} k_{2 \perp}^{b}\right) \\
& \times\left[\frac{-k_{1 \perp}^{\mu_{1}}}{\boldsymbol{k}_{1 \perp}^{2}}\left(\Pi_{\|}^{\mu_{2} \mu_{3}}\left(k_{2 \|}\right)-\Pi_{\|}^{\mu_{2} \mu_{3}}\left(k_{3 \|}\right)\right)+\frac{k_{1 \perp}^{\mu_{1}} k_{2 \perp}^{\mu_{2}}-k_{1 \perp}^{\mu_{2}} k_{2 \perp}^{\mu_{1}}-\left(\boldsymbol{k}_{1} \cdot \boldsymbol{k}_{2}\right)_{\perp} g_{\perp}^{\mu_{1} \mu_{2}}}{\boldsymbol{k}_{1 \perp}^{2} \boldsymbol{k}_{2 \perp}^{2}} k_{2 \| \nu} \Pi_{\|}^{\mu_{3} \nu}\left(k_{3 \|}\right)\right. \\
& \left.+ \text { permutations of }\left(k_{1}, \mu_{1}\right),\left(k_{2}, \mu_{2}\right) \text {, and }\left(k_{3}, \mu_{3}\right)\right] \text {, } \\
& T_{H L L 2}^{\mu_{1} \mu_{2} \mu_{3}}\left(k_{1}, k_{2}, k_{3}\right)=-\frac{2 e}{i} \operatorname{sign}(e B)
\end{aligned}
$$

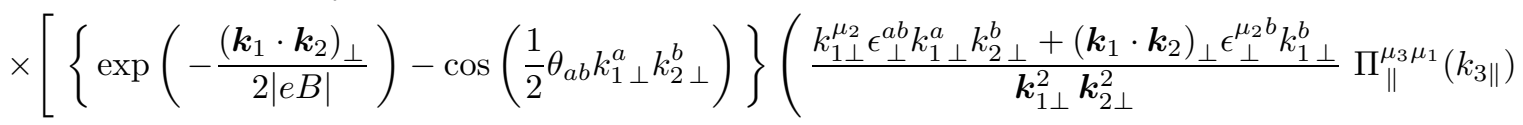

$$
\begin{aligned}
& \left.+\frac{k_{2 \perp}^{\mu_{1}} \epsilon_{\perp}^{a b} k_{2 \perp}^{a} k_{1 \perp}^{b}+\left(\boldsymbol{k}_{2} \cdot \boldsymbol{k}_{1}\right)_{\perp} \epsilon_{\perp}^{\mu_{1} b} k_{2 \perp}^{b}}{\boldsymbol{k}_{1 \perp}^{2} \boldsymbol{k}_{2 \perp}^{2}} \Pi_{\|}^{\mu_{2} \mu_{3}}\left(k_{3 \|}\right)+\frac{g_{\perp}^{\mu_{1} \mu_{2}} \epsilon_{\perp}^{a b} k_{1 \perp}^{a} k_{2 \perp}^{b}+\epsilon_{\perp}^{\mu_{1} \mu_{2}}\left(\boldsymbol{k}_{1} \cdot \boldsymbol{k}_{2}\right)_{\perp}}{\boldsymbol{k}_{1 \perp}^{2} \boldsymbol{k}_{2 \perp}^{2}} k_{2 \| \nu} \Pi_{\|}^{\mu_{3} \nu}\left(k_{3 \|}\right)\right) \\
& \left.+ \text { permutations of }\left(k_{1}, \mu_{1}\right),\left(k_{2}, \mu_{2}\right) \text {, and }\left(k_{3}, \mu_{3}\right)\right] \text {. }
\end{aligned}
$$

Here we defined $(\boldsymbol{p} \cdot \boldsymbol{q})_{\perp}=p^{1} q^{1}+p^{2} q^{2}$. These contributions come from the HLL terms in Eqs. (21) and (22). The HLL contribution coming from $\Delta_{H L L}^{\mu_{\|} \nu_{\|} \lambda_{\|}}$in Eq. (20) is of a higher order in $1 /|e B|$ and therefore is neglected here. ${ }^{3}$

Using Eqs. (18), (25), and (26), one can easily check that the 3-point vertex $T^{\mu_{1} \mu_{2} \mu_{3}}$ is transverse. The cancellation occurs between $T_{L L L}^{\mu_{1} \mu_{2} \mu_{3}}$ and $T_{H L L 1}^{\mu_{1} \mu_{2} \mu_{3}}$. As to the term $T_{H L L 2}^{\mu_{1} \mu_{2} \mu_{3}}$, it is transverse itself.

Is there a kinematic region in which the LLL contribution is dominant? The answer to this question is "yes". It is the region with momenta $\boldsymbol{k}_{i \perp}^{2} \gg\left|k_{i \|}^{2}\right|$. In this case, the leading terms in the expansion of the LLL and HLL vertices in powers of $k_{i \|}$ are:

$$
\begin{array}{r}
T_{L L L}^{\mu_{1} \mu_{2} \mu_{3}}\left(k_{1}, k_{2}, k_{3}\right)=-\frac{2 e \tilde{\alpha}}{3 \pi} \frac{|e B|}{m^{2}} \sin \left(\frac{1}{2} \theta_{a b} k_{1 \perp}^{a} k_{2 \perp}^{b}\right)\left[\left(k_{2}-k_{3}\right)_{\|}^{\mu_{1}} g_{\|}^{\mu_{2} \mu_{3}}+\left(k_{3}-k_{1}\right)_{\|}^{\mu_{2}} g_{\|}^{\mu_{3} \mu_{1}}+\left(k_{1}-k_{2}\right)_{\|}^{\mu_{3}} g_{\|}^{\mu_{1} \mu_{2}}\right], \\
T_{H L L 1}^{\mu_{1} \mu_{2} \mu_{3}}\left(k_{1}, k_{2}, k_{3}\right)=-\frac{2 e \tilde{\alpha}}{3 \pi} \frac{|e B|}{m^{2}} \sin \left(\frac{1}{2} \theta_{a b} k_{1 \perp}^{a} k_{2 \perp}^{b}\right)\left[\frac{-k_{1 \perp}^{\mu_{1}}}{\boldsymbol{k}_{1 \perp}^{2}}\left\{\left(k_{2 \|}^{2}-k_{3 \|}^{2}\right) g_{\|}^{\mu_{2} \mu_{3}}-k_{2 \|}^{\mu_{2}} k_{2 \|}^{\mu_{3}}+k_{3 \|}^{\mu_{2}} k_{3 \|}^{\mu_{3}}\right\}\right. \\
+\frac{k_{1 \perp}^{\mu_{1}} k_{2 \perp}^{\mu_{2}}-k_{1 \perp}^{\mu_{2}} k_{2 \perp}^{\mu_{1}}-\left(\boldsymbol{k}_{1} \cdot \boldsymbol{k}_{2}\right)_{\perp} g_{\perp}^{\mu_{1} \mu_{2}}}{\boldsymbol{k}_{1 \perp}^{2} \boldsymbol{k}_{2 \perp}^{2}}\left\{k_{3 \|}^{2} k_{2 \|}^{\mu_{3}}-\left(k_{2} \cdot k_{3}\right) \| k_{3 \|}^{\mu_{3}}\right\} \\
\left.+\operatorname{permutations~of~}\left(k_{1}, \mu_{1}\right),\left(k_{2}, \mu_{2}\right), \text { and }\left(k_{3}, \mu_{3}\right)\right],
\end{array}
$$

\footnotetext{
3 Note that the vertex for the initial non-smeared fields $A_{\mu}$ is given by $e^{-\frac{\boldsymbol{k}_{1 \perp}^{2}+\boldsymbol{k}_{2 \perp}^{2}+\boldsymbol{k}_{3 \perp}^{2}}{4|e B|}} T^{\mu_{1} \mu_{2} \mu_{3}}$. Thus, for these fields, an exponentially damping form-factor occurs not in the propagator but in their vertices (compare with a discussion of this feature in Ref. [5]).
} 


$$
\begin{aligned}
& T_{H L L 2}^{\mu_{1} \mu_{2} \mu_{3}}\left(k_{1}, k_{2}, k_{3}\right)=\frac{2 e \tilde{\alpha}}{3 \pi} \frac{e B}{m^{2}}[\left.\exp \left(-\frac{\left(\boldsymbol{k}_{1} \cdot \boldsymbol{k}_{2}\right)_{\perp}}{2|e B|}\right)-\cos \left(\frac{1}{2} \theta_{a b} k_{1 \perp}^{a} k_{2 \perp}^{b}\right)\right\} \\
& \times\left(\frac{k_{1 \perp}^{\mu_{2}} \epsilon_{\perp}^{a b} k_{1 \perp}^{a} k_{2 \perp}^{b}+\left(\boldsymbol{k}_{1} \cdot \boldsymbol{k}_{2}\right)_{\perp} \epsilon_{\perp}^{\mu_{2} b} k_{1 \perp}^{b}}{\boldsymbol{k}_{1 \perp}^{2} \boldsymbol{k}_{2 \perp}^{2}}\left\{k_{3 \|}^{2} g_{\|}^{\mu_{3} \mu_{1}}-k_{3 \|}^{\mu_{3}} k_{3 \|}^{\mu_{1}}\right\}\right. \\
&+\frac{k_{2 \perp}^{\mu_{1}} \epsilon_{\perp}^{a b} k_{2 \perp}^{a} k_{1}^{b}+\left(\boldsymbol{k}_{2} \cdot \boldsymbol{k}_{1}\right)_{\perp} \epsilon_{\perp}^{\mu_{1} b} k_{2 \perp}^{b}}{\boldsymbol{k}_{1 \perp}^{2} \boldsymbol{k}_{2 \perp}^{2}}\left\{k_{3 \|}^{2} g_{\|}^{\mu_{2} \mu_{3}}-k_{3 \|}^{\mu_{2}} k_{3 \|}^{\mu_{3}}\right\} \\
&\left.+\frac{g_{\perp}^{\mu_{1} \mu_{2}} \epsilon_{\perp}^{a b} k_{1 \perp}^{a} k_{2 \perp}^{b}+\epsilon_{\perp}^{\mu_{1} \mu_{2}}\left(\boldsymbol{k}_{1} \cdot \boldsymbol{k}_{2}\right)_{\perp}}{\boldsymbol{k}_{1 \perp}^{2} \boldsymbol{k}_{2 \perp}^{2}}\left\{k_{3 \|}^{2} k_{2 \|}^{\mu_{3}}-\left(k_{2} \cdot k_{3}\right) k_{3 \|}^{\mu_{3}}\right\}\right) \\
&\left.+\operatorname{permutations~of~}\left(k_{1}, \mu_{1}\right),\left(k_{2}, \mu_{2}\right), \text { and }\left(k_{3}, \mu_{3}\right)\right] .
\end{aligned}
$$

It is clear from these expressions that in that region the LLL contribution dominates indeed. ${ }^{4}$ This result is quite noticeable. The point is that as was shown in Ref. [7], the region with momenta $\boldsymbol{k}_{i \perp}^{2} \gg\left|k_{i \|}^{2}\right|$ yields the dominant contribution in the Schwinger-Dyson equation for the dynamical fermion mass in QED in a strong magnetic field. Therefore the LLL approximation is reliable in that problem.

Nondecoupling of (heavy) HLL in the infrared region is quite unexpected phenomenon. What physics underlines it? We believe that this phenomenon reflects the important role of a boundary dynamics at spatial infinity in this problem. The point is that the HLL are not only heavy states but their transverse size grows without limit with their gap $\sqrt{m^{2}+2|e B| n}$ as $n \rightarrow \infty$. This happens because the transverse dynamics in the Landau problem is oscillator-like one. Since in order to cancel the LLL anomaly one should consider the contribution of all higher Landau levels, it implies that the role of the boundary dynamics at the transverse spatial infinity (corresponding to $n \rightarrow \infty$ ) is crucial for the restoration of the gauge symmetry. In this respect this phenomenon is similar to that of edge states in the quantum Hall effect: the edge states are created by the boundary dynamics and also restore the gauge invariance [1]. Both these phenomena reflect the importance of a boundary dynamics in a strong magnetic field. It would be interesting to examine whether similar nondecoupling phenomena take place in noncommutative theories arising in string theories in magnetic backgrounds [4].

\section{Acknowledgments}

Discussions with A. Buchel, V. Gusynin, and I. Shovkovy are acknowledged. We are grateful for support from the Natural Sciences and Engineering Research Council of Canada.

[1] W. Heisenberg and H. Euler, Z. f. Phys. 98, 714 (1936); V. F. Weisskopf, Kong. Dansk. Vid. Sels. Mat.-Fys. Medd. XIV, No. 6 (1936).

[2] J. Schwinger, Phys. Rev. 82, 664 (1951).

[3] G. V. Dunne, hep-th/0406216

[4] M. R. Douglas and N. A. Nekrasov, Rev. Mod. Phys. 73, 977 (2001); R. J. Szabo, Phys. Rep. 378, 207 (2003).

[5] E. V. Gorbar and V. A. Miransky, Phys. Rev. D 70, 105007 (2004).

[6] S. Minwalla, M. Van Raamsdonk and N. Seiberg, JHEP 0002, 020 (2000).

[7] V. P. Gusynin, V. A. Miransky, and I. A. Shovkovy, Phys. Rev. Lett. 83, 1291 (1999); Nucl. Phys. B563, 361 (1999); Phys. Rev. D 67, 107703 (2003).

[8] A. Chodos, K. Everding, and D. A. Owen, Phys. Rev. D 42, 2881 (1990); V. P. Gusynin, V. A. Miransky, and I. A. Shovkovy, Nucl. Phys. B462, 249 (1996).

[9] G. Passarino and M. J. G. Veltman, Nucl. Phys. B160, 151 (1979).

\footnotetext{
${ }^{4}$ Although the vertex $T_{H L L 1}^{\mu_{1} \mu_{2} \mu_{3}}$ is subdominant in this region, it is crucial for the restoration of the Ward identity. It is because while in the Ward identity there are terms in the HLL vertex $T_{H L L 1}^{\mu_{1} \mu_{2} \mu_{3}}$ which are multiplied by a large transverse momentum $k_{\perp}$, the LLL vertex $T_{L L L}^{\mu_{1} \mu_{2} \mu_{3}}$ is multiplied only by a small longitudinal momentum $k_{\|}$.
} 
[10] I. S. Gradshtein and I. M. Ryzhik, Table of Integrals, Series and Products (Academic Press, New York, 2000).

[11] X. G. Wen, Phys. Rev. Lett. 64, 2206 (1990). 